\title{
Hypoxic Ischemic Encephalopathy
}

National Cancer Institute

\section{Source}

National Cancer Institute. Hypoxic Ischemic Encephalopathy. NCI Thesaurus. Code C35549.

Ischemic brain damage in which the entire brain is deprived of oxygen. It may be fatal or lead to long term disabilities including developmental delays, intellectual disability, and seizures. 